\title{
Actitudes de madres adultas y adolescentes hacia la crianza de bebés prematuros
}

\author{
Beatriz Villamizar Carvajal² \\ Carolina Vargas Porras 3 \\ Eugenia Rueda Nieves ${ }^{4}$
}

doi:10.11144/Javeriana.ie19-2.amaa

Cómo citar: Villamizar Carvajal B, Vargas Porras C, Rueda Nieves E. Actitudes de madres adultas y adolescentes hacia la crianza de bebés prematuros. Investig Enferm Imagen Desarr. 2017;19(2):41-52. http://dx.doi.org/10.11144/Javeriana.ie19-2.amaa

1. Artículo original de investigación. Fecha de recepción: 4 de noviembre de 2015. Fecha de aceptación: 16 de mayo de 2016.

2. Doctora en Enfermería. Profesora de la Escuela de Enfermería, Facultad de Salud, Universidad Industrial de Santander, Bucaramanga, Colombia. Correo electrónico: beatriz@ uis.edu.co

3. Magíster en Enfermería Materno Perinatal. Profesora de la Escuela de Enfermería, Facultad de Salud, Universidad Industrial de Santander, Bucaramanga, Colombia. Correo electrónico: cvargasporras@yahoo.com

4. Especialista en Cuidado Crítico. Profesora de la Escuela de Enfermería, Facultad de Salud, Universidad Industrial de Santander, Bucaramanga, Colombia. Correo electrónico: efirueda@hotmail.com 


\section{Resumen}

Introducción: Un nacimiento prematuro trae consigo la interrupción de la temprana relación madre-hijo, que influye en la formación del apego o en las actitudes hacia la crianza y se convierten en una situación de especial vulnerabilidad. Objetivo: Determinar las actitudes de madres adultas y adolescentes hacia la crianza de bebés prematuros. Método: Estudio de corte transversal, con muestra no probabilística de 204 madres de bebés prematuros. La información se recolectó entre agosto y diciembre del 2013 en Bucaramanga, a través del Inventario de Paternidad para Adultos y Adolescentes, conformado por cuatro dimensiones relacionadas con las expectativas inadecuadas, falta de empatía, la creencia en el castigo físico y la inversión del rol materno, que miden el riesgo de negligencia en el cuidado. Se utilizó el programa Stata versión $12 \mathrm{R}$, para el procesamiento y análisis de la información. Resultados: Las madres tanto adolescentes como adultas no mostraron diferencias estadísticamente significativas en la puntuación de las dimensiones expectativas inadecuadas $(p=0,97)$, creencia en el castigo físico $(p=0,34)$, inversión del rol materno $(p=0,68)$, las cuales se ubicaron en calificaciones promedias y altas, pero en la dimensión falta de empatía sí hubo diferencia significativa $(p=0,02)$. La mayoría de madres presentaron un riesgo mínimo de negligencia en el cuidado. Conclusiones: Al parecer, la condición de prematurez hace que estos dos grupos de madres no tengan diferencias significativas en sus actitudes hacia la crianza, pues, en general, son adecuadas, a pesar de la vivencia de la hospitalización con mucha carga de estrés.

Palabras clave: actitudes; madres; prematuro; crianza

\section{Attitudes of Adult and Teenage Mothers towards Premature Parenting}

\section{Abstract}

Introduction: A preterm birth entails the disruption of early mother-child relationship, which influences the formation of attachment, attitudes toward parenting and their becoming particularly vulnerable. Objective: To determine attitudes from adult and teenage mothers raising preemies. Method: Cross-sectional study with nonrandom sample of 204 mothers of premature babies. The information was collected between August and December 2013 in Bucaramanga, through the Inventory of Parenting for Adults and Adolescents, it was made up of four dimensions related to inappropriate expectations, lack of empathy, belief in corporal punishment and the reversal of the maternal role, which measure the risk of negligence in care. The program that was used for processing and analyzing information was Stata version 12R. Results: Both mothers, adolescents and adults did not show statistically significant differences in relation to the score on inappropriate expectations $(p=0.97)$, belief in corporal punishment $(p=0.34)$, maternal role reversal $(p=0,68)$, which ranged between average and high scores, but regarding the dimension of lack of empathy, there was in deed a significant difference $(p=0.02)$. Most mothers had a minimal risk of negligence in care. Conclusions: Apparently, the condition of prematurity does not cause these two groups of mothers to have significant differences in their attitudes towards parenting, as they are generally adequate, despite the experience of hospitalization and the heavy load of stress.

Keywords: attitudes; mothers; premature; parenting 


\section{Atitudes de mães adultas e adolescentes em relação à criação dos bebês prematuros}

\section{Resumo}

Introdução: Um nascimento prematuro implica interrupção do precoce relacionamento mãe-filho, influenciando a formação de apego no que diz respeito das atitudes para a criação, que tornam a situação em uma de especial vulnerabilidade. Objetivo: Determinar as atitudes de mães adultas e mães adolescentes para a criação de bebés prematuros. Método: Estudo de tipo transversal com amostra não probabilística de 204 mães de bebés prematuros. A informação foi coletada entre agosto e dezembro de 2013 em Bucaramanga, através do Inventário de Paternidade para Adultos e Adolescentes, conformado por quatro dimensões relacionadas com as expectativas inadequadas, falta de empatia, crença no castigo físico e inversão do papel materno, que medem o risco de negligenciar o cuidado. O programa Stata versão $12 \mathrm{R}$ foi utilizado para processamento e análise de informações. Resultados: As mães tanto adolescentes quanto adultas não mostraram diferenças estatisticamente significativas na pontuação das dimensões expetativas inadequadas ( $\mathrm{p}=0,97$ ), crença no castigo físico $(p=0,34)$, inversão do papel materno $(p=0,68)$, as quais se situaram em calcificações médias e altas, mas na dimensão falta de empatia se houve diferencia significativa $(\mathrm{p}=0,02)$. A maioria de mães apresentou risco mínimo de negligenciar o cuidado. Conclusões: Aparentemente, a condição de prematuração faz com que estes dois grupos de mães não mostraram diferenças significativas nas suas atitudes para a criação, pois, em geral, são adequadas, ainda trás a vivencia da hospitalização com pesada carga de estrese.

Palavras-chave: atitudes; mães; prematuração; criação 


\section{Introducción}

La prematurez es un problema de salud perinatal importante en todo el mundo y se estima que cada año nacen unos 15 millones de niños prematuros (1), lo cual trae consigo una interrupción de la relación temprana madrehijo que influye en la formación del apego y en las actitudes hacia la crianza. Diversas condiciones de la hospitalización en las unidades de cuidados intensivo neonatales (UCIN) no permiten que la madre asuma su rol, lo que hace difícil la crianza inicial, y la enorme demanda tanto en términos económicos como en emocionales convierte esta experiencia en un periodo crítico de alta vulnerabilidad para presentar distrés agudo, síntomas de ansiedad y depresión (2).

Desde 1999, la psicóloga Mary Ainsworth planteó que la unión madre e hijo difiere en la calidad de sus relaciones de apego. Asimismo, existe evidencia sobre la relación temprana madre-hijo como uno de los factores que exacerban o reducen los efectos potencialmente adversos del nacimiento prematuro (3).

En los últimos años se han realizado importantes esfuerzos en las UCIN para crear condiciones favorables tendientes a permitir que las madres permanezcan cerca y realicen actividades con su hijo para fortalecer el vínculo de la díada madre-hijo. A pesar de ello, es frecuente que se presenten problemas en esta interacción, relacionados con la prematuridad, y que estos persistan al menos durante el primer año de vida. De hecho, las madres de estos niños suelen mostrar ansiedad y escasa confianza en sus habilidades como cuidadoras, debido a la falta de orientación sobre los cuidados con niños prematuros (4).

Como estos niños prematuros tienen problemas para procesar la información y, en consecuencia, su comportamiento se desorganiza con facilidad, los hace parecer poco reactivos frente a los estímulos cotidianos, por lo que las madres tienden a compensar este hecho ya sea asumiéndolo como algo normal o sobrestimulándolos. Esto, entonces, genera un efecto no deseado de desorganizar todavía más el comportamiento del niño, al pasar de la hipoactividad a la hiperactividad de una forma abrupta (5). Todo ello desorienta al cuidador sobre las pautas de crianza apropiadas para un bebé con este perfil conductual (6).

Por lo ya expuesto, es necesario determinar las actitudes hacia la crianza de bebés prematuros en madres adultas y adolescentes, para aportar a las diferentes intervenciones que se puedan aplicar a estas madres en el fortalecimiento de las conductas parentales positivas desde la etapa temprana del nacimiento (7).

\section{Método}

Estudio descriptivo de corte transversal, realizado en Bucaramanga (Colombia), en el periodo agosto-diciembre de 2013. La población estuvo conformada por madres de bebés prematuros nacidos en una institución de tercer nivel de atención. La muestra fue no probabilística, y estuvo conformada por 204 madres de prematuros.

44 Los criterios de inclusión fueron: mujeres multíparas que se encontraran entre el día 1 y los 14 meses después del parto; mujeres primíparas 
que tenían, por lo menos, entre 8 días posparto y 14 meses; y madres con hijos prematuros (edad gestacional menor de 37 semanas). Los criterios de exclusión fueron: mujeres con óbito fetal o mortinato, mujeres con alteraciones de la comunicación, mujeres con enfermedades psiquiátricas diagnosticadas y mujeres consumidoras de sustancias psicoactivas que generan alteración de la conciencia.

Las variables estuvieron medidas por el Inventario de Paternidad para Adultos y Adolescentes (IPAA), el cual cuenta con una adecuada consistencia interna según el alfa de Cronbach y validez diagnóstica y discriminante (8), que mide cuatro dimensiones con una escala tipo Likert (cinco opciones de respuesta, que van desde totalmente de acuerdo hasta totalmente en desacuerdo), conformada por 32 items. Las dimensiones son: 1) expectativas inapropiadas hacia los niños, que se refiere a las demandas de los padres hacia los hijos; 2) falta de empatía hacia las necesidades del niño, que se refiere a la inhabilidad para ser conscientes de las necesidades de los niños y responder a ellas de una manera apropiada; 3) creencia en el valor del castigo físico, que se refiere a la fuerte creencia en el valor del castigo físico para formar a los niños, y 4) inversión del rol madre-hijo, que se refiere a la creencia de que los niños deben ser sensibles ante las necesidades emocionales de los padres. Este inventario mide las actitudes hacia la crianza que se consideran de alto riesgo, que favorecen la negligencia (forma particular de maltrato infantil, causada por descuido u omisión) o el abuso infantil hacia los niños, lo cual permite visualizar, a su vez, las actitudes que la madre puede tener en el cuidado de su hijo prematuro.

Para el análisis de la información se siguieron los parámetros según el instructivo del IPAA, sumando los ítems y convirtiéndolos a puntuaciones específicas para determinar los decatipos, según lo establecido en madres adolescentes y adultas, con la correspondiente interpretación así: puntaje bajo, de 1 a 4, que equivale a deficiencias en la conducta materna adecuada; puntaje promedio, de 5 a 6 , que equivale a la conducta materna estándar; y puntaje alto, de 7 a 10, que equivale a actitudes positivas hacia la crianza.

$\mathrm{El}$ instrumento se aplicó en un solo momento mediante una entrevista personalizada. La información se recolectó durante cinco meses consecutivos en los servicios de hospitalización de puerperio, en consulta externa y a nivel domiciliario.

Se elaboró la correspondiente base de datos en Excel, que conservó la codificación de los instrumentos para mantener la confidencialidad de los informantes. Se utilizó el programa Stata versión 12R para el procesamiento, y el análisis de la información se hizo según el instructivo del IPAA para determinar las calificaciones en las dimensiones tanto para el grupo de madres adolescentes como para el grupo de madres adultas, y los dos grupos se compararon mediante la prueba estadística t de Student, pues los datos presentaron una distribución normal. El instructivo del IPAA define una puntuación diferente para las madres adultas y adolescentes, y por eso se dividen los dos grupos de análisis.

Se siguieron los lineamientos de la Resolución 008430 de 1993 (9), en la cual se establecen las normas científicas, técnicas y administrativas 
para la investigación en salud. Según esta resolución, se clasifica como una investigación sin riesgo. El artículo 37 dice: "las investigaciones en mujeres durante el puerperio se permitirán cuando no interfieran con la salud de la madre y ni con la del recién nacido". Se obtuvo el correspondiente aval del Comité de Ética en Investigación Científica de la Universidad Industrial de Santander, según acta 03 del 2013 y se consideraron las pautas éticas internacionales para la investigación biomédica en seres humanos recomendadas por el Consejo de Organizaciones Internacionales de las Ciencias Médicas (10). Se solicitó la firma del consentimiento o asentimiento informado escrito, según correspondiera.

\section{Resultados}

Participaron 204 mujeres que, en su mayoría, fueron adultas con un promedio de edad de 26 años, tanto multíparas como primíparas. En su mayoría, la vía del parto fue cesárea, como se puede observar en la tabla 1.

TABLA 1. Características sociodemográficas

\begin{tabular}{|c|c|}
\hline \multicolumn{2}{|c|}{ Caracteristicas sociodemográficas de las madres } \\
\hline Edad de la madre & Porcentaje \\
\hline Adolescentes & 12,7 \\
\hline Adultas & 87,3 \\
\hline Paridad & Porcentaje \\
\hline Primiparas & 47,1 \\
\hline Multíparas & 52,9 \\
\hline Via del parto & Porcentaje \\
\hline Vaginal & 25,9 \\
\hline Cesárea & 74,1 \\
\hline Estrato socioeconómico & Porcentaje \\
\hline 1 & 56,8 \\
\hline 2 & 33,5 \\
\hline 3 & 9,3 \\
\hline 4 & 0,4 \\
\hline Ocupación & Porcentaje \\
\hline Trabaja & 32,3 \\
\hline Estudia & 3,4 \\
\hline Ama de casa & 64,3 \\
\hline Nivel educativo & Porcentaje \\
\hline Primaria incompleta & 5,4 \\
\hline Primaria completa & 17,6 \\
\hline
\end{tabular}




\begin{tabular}{|l|r|r|}
\hline Bachillerato incompleto & 26,4 \\
\hline Bachillerato completo & 44,3 \\
\hline Técnico o tecnólogo & 3,4 \\
\hline Universitario & 2,9 \\
\hline \multicolumn{2}{|c|}{ Caracteristicas de los hijos } \\
\hline Minima gestacional & Semanas \\
\hline Máxima & & 26 \\
\hline Promedio Dias de nacimiento & 36 \\
\hline & & 33 \\
\hline Minima & Dias \\
\hline Máxima & & $1 / 7$ \\
\hline Promedio & & 420 \\
\hline
\end{tabular}

Fuente: elaboración propia.

Al clasificar a las madres adolescentes y madres adultas según el puntaje en cada dimensión de acuerdo con el instructivo del IPAA, se estableció que existe un riesgo mínimo de negligencia en el cuidado, esto teniendo en cuenta que el puntaje, en general, se ubicó en calificaciones promedias y altas, como se puede observar en la tabla 2 del perfil de dimensiones del IPAA.

Tabla 2. Perfil de dimensiones del IPAA en madres adolescentes y adultas (n [\%])

\begin{tabular}{|c|c|c|}
\hline Dimensiones & $\begin{array}{c}\text { Adolescentes } \\
(\mathrm{n}=26)\end{array}$ & $\begin{array}{l}\text { Adultas } \\
(\mathrm{n}=178)\end{array}$ \\
\hline \multicolumn{3}{|c|}{ Dimensión A: Expectativas inapropiadas hacia los niños } \\
\hline Calificación baja: 1-4 & $4(15,3)$ & $29(16,3)$ \\
\hline Calificación promedio: $5-6$ & $10(38,5)$ & $62(34,8)$ \\
\hline Calificación alta: $7-10$ & $12(46,2)$ & $87(48,9)$ \\
\hline \multicolumn{3}{|c|}{ Dimensión B: Falta de empatia hacia las necesidades del niño } \\
\hline Calificación baja: 1-4 & $5(19,2)$ & $51(28,6)$ \\
\hline Calificación promedio: $5-6$ & $9(34,6)$ & $70(39,3)$ \\
\hline Calificación alta: $7-10$ & $12(46,2)$ & $57(32,1)$ \\
\hline \multicolumn{3}{|c|}{ Dimensión C: Creencia en el valor del castigo físico } \\
\hline Calificación baja: 1-4 & $4(15,4)$ & $33(18,5)$ \\
\hline Calificación promedio: $5-6$ & $3(11,5)$ & $28(15,7)$ \\
\hline Calificación alta: $7-10$ & $19(73,1)$ & $117(65,8)$ \\
\hline \multicolumn{3}{|c|}{ Dimensión D: Inversión del rol madre-hijo } \\
\hline Calificación baja: 1-4 & $8(30,75)$ & $66(37,0)$ \\
\hline Calificación promedio: 5-6 & $10(38,5)$ & $57(32,1)$ \\
\hline Calificación alta: 7-10 & $8(30,75)$ & $55(30,9)$ \\
\hline
\end{tabular}

Fuente: elaboración propia. 
De esta forma, el perfil de dimensiones reportó que tanto las madres adolescentes como las adultas, en su gran mayoria, se ubicaron en calificaciones promedias y altas, es decir, reflejan lo estándar esperado, donde las madres anhelan un desarrollo adecuado del niño, comprenden las necesidades del niño, creen en otras estrategias para disciplinar y presentan un rol adecuado como madres. Sin embargo, algunas madres presentaron calificaciones bajas en las diferentes dimensiones del IPAA, lo que refleja deficiencias en la conducta materna adecuada y riesgo de negligencia en el cuidado de los hijos de estas madres, y a pesar de haber sido poco evidenciado, se debe dar la importancia que esto representa para las actitudes hacia la crianza de los recién nacidos prematuros.

Para la comparación de las madres adolescentes y adultas se establecieron las medias y las desviaciones estándar de los puntajes totales decatipo por cada una de las dimensiones, sin mostrar diferencias estadísticamente significativas entre ellas, como se observa en la tabla 3. Pese a que en la dimensión "falta de empatía hacia las necesidades del niño" señala una leve diferencia estadística, es de resaltar que la media de los puntajes en los dos grupos corresponde a calificaciones que se interpretan como actitudes adecuadas para la crianza.

TABLA 3. Dimensiones del IPAA en madres adolescentes y adultas según puntaje decatipo

\begin{tabular}{|l|r|r|r|r|}
\hline \multicolumn{1}{|c|}{ Dimensiones } & $\begin{array}{c}\text { Adolescentes } \\
\text { Media (DE) }\end{array}$ & $\begin{array}{c}\text { Adultas } \\
\text { Media (DE) }\end{array}$ & $\begin{array}{c}\text { t de } \\
\text { Student }\end{array}$ & Valor p \\
\hline $\begin{array}{l}\text { Dimensión A: Expectativas } \\
\text { inapropiadas hacia los niños }\end{array}$ & $6,38(2,22)$ & $6,37(1,83)$ & 0,04 & 0,97 \\
\hline $\begin{array}{l}\text { Dimensión B: Falta de em- } \\
\text { patía hacia las necesidades } \\
\text { del niño }\end{array}$ & $6,77(1,85)$ & $5,51(2,61)$ & 2,37 & 0,02 \\
\hline $\begin{array}{l}\text { Dimensión C: Creencia en el } \\
\text { valor del castigo físico }\end{array}$ & $7,27(2,3)$ & $6,78(2,44)$ & 0,96 & 0,34 \\
\hline $\begin{array}{l}\text { Dimensión D: Inversión del } \\
\text { rol madre-hijo }\end{array}$ & $5,19(2,56)$ & $4,99(2,26)$ & 0,42 & 0,68 \\
\hline
\end{tabular}

Fuente: elaboración propia.

\section{Discusión}

En el análisis de los resultados de las cuatro dimensiones del IPAA se observó que las madres adolescentes y madres adultas de prematuros tienen expectativas apropiadas hacia los niños. Este resultado es distinto a lo señalado años atrás en la literatura, donde se mencionaba que cuando el bebé es prematuro, su apariencia no corresponde a las expectativas maternas "asociadas a un bebé nacido a término y saludable, para iniciar secuencias comportamentales recíprocas y, por ende, la formación del vínculo puede demorarse porque él bebé no es hábil para desempeñar su parte en el establecimiento del apego" (11).

Además, en este estudio los hallazgos evidencian que tanto las madres adolescentes como las madres adultas de bebés prematuros, en su mayoria, tienen empatía hacia las necesidades del niño; pero es importante reflexionar 
sobre aquellas madres que presentan falta de empatía hacia las necesidades del niño. Un estudio (12) señaló que las madres adultas, a diferencia de las adolescentes, suelen sintonizar mejor con las señales y demandas de sus bebés en algunas situaciones de cuidado. Las interacciones de las madres adolescentes con sus bebés parecen caracterizarse por estar menos organizadas. La adopción del rol materno en las madres primerizas adolescentes está determinada por la presencia de la pareja, como un agente positivo para que dicho rol se establezca de forma favorable, al compartir responsabilidades en relación con la crianza del bebé y por el afecto y seguridad que se da al convivir los dos solos. Las madres adolescentes, que viven solo con su familia de origen, evidencian resultados opuestos y alcanzan los niveles más bajos de adopción de rol materno, al desarrollarse una hiperprotección de los padres hacia ellas y su bebé. Dicho factor impide que esta realice las actividades necesarias para adoptar adecuadamente el rol de madre y se configura un futuro incierto en la relación "madre-hijo" (13).

En los resultados de este estudio se evidenció que las madres adolescentes y las madres adultas de bebés prematuros, en la dimensión relacionada con la creencia en el valor del castigo físico, presentaron puntajes bajos. De igual manera, otras investigaciones $(14,15)$ han mostrado que no existe preferencia en el castigo físico, sino diferentes formas de disciplinar; se mencionan factores que influyen en elegir el tipo de disciplina, como lo son el nivel educativo de la madre, la situación laboral y el ingreso familiar. Por el contrario, existen estudios $(16,17)$ que mencionan la creencia en el castigo físico para corregir una mala conducta, lo que muestra complicaciones a futuro de los hijos con el uso de este tipo de disciplina punitiva. También se han encontrado algunos factores presentes en la madre, como son la depresión y el estrés, asociados al uso del castigo físico $(18,19)$; pero aún la literatura no reporta hallazgos específicamente en madres de bebés prematuros.

Así mismo, las madres de bebés prematuros tienen sentimientos de culpa y decepción, que llevan a la depresión y, por ende, dificultan la interacción con su hijo (20), debido a que deben iniciar la maternidad de manera inesperada y no tienen control sobre la situación, por lo que se sienten frustradas e inseguras en el rol materno (21-23). Las madres angustiadas pueden desarrollar pautas de crianza restrictivas, que generan un impacto negativo en el desarrollo del bebé (24). Igualmente, las madres adolescentes tienen riesgo social, porque presentan menor capacidad de regulación emocional con sus hijos y mayor probabilidad de utilizar estrategias disciplinarias de maltrato (25).

Por último, el hallazgo de este estudio con relación al rol materno llama la atención, dado que el porcentaje que presenta inversión del rol madrehijo es similar tanto en madres adolescentes como en madres adultas. El nacimiento del bebé en condición de prematuridad o bajo peso genera cierto grado de ruptura en la forma de crianza y en la distribución de las tareas entre el padre y la madre (26). La actitud protectora por el hijo se relaciona con el proceso de la adopción del rol materno, que la hace sentir y actuar diferente con mayor responsabilidad hacia el nuevo ser; se proyecta al futuro con una orientación de su accionar hacia la satisfacción de las necesidades 
físicas y emocionales de su hijo prematuro, a quien lo percibe como un niño en riesgo, frágil y diferente de los otros (27). Todo lo anterior es el resultado de la trayectoria de la hospitalización del prematuro en la cual las madres están dispuestas a aprender a cuidar a su hijo, con el fin de alcanzar su independencia como cuidadoras y, de esta manera, fortalecer el vínculo afectivo para el desempeño del rol materno (28).

\section{Conclusiones}

La literatura refiere comportamientos diferentes en el desempeño del rol materno entre madres adultas y adolescentes, pero este estudio no reportó diferencias significativas entre ellas. Al parecer, la condición de prematurez hace que estos dos grupos de madres no tengan diferencias significativas en sus actitudes hacia la crianza, evidenciadas en las dimensiones relacionadas con las expectativas hacia el niño, la empatía hacia las necesidades del niño, la creencia en el valor del castigo físico y la inversión del rol madrehijo, pues, en general, son adecuadas, a pesar de la vivencia de la hospitalización con mucha carga de estrés.

\section{Financiación}

Este artículo es producto del proyecto con código 5697, financiado por la Vicerrectoría de Investigación y Extensión de la Universidad Industrial de Santander, Colombia.

\section{Conflictos de interés}

Las autoras declaramos que no tenemos conflictos de interés.

\section{Referencias}

1. Beck S, Wojdyla D, Say L, Betrán AP, Merialdi M, Harris J, et al. The worldwide incidence of preterm birth: a systematic review of maternal mortality and morbidity. Bull World Health Organ. 2010;88:31-8.

2. Jubinville J, Newburn-Cook C, Hegadoren K, Lacaze-Masmonteil T. Symptoms of acute stress disorder in mothers of premature infants. Adv Neonatal Care. 2012;12(4):246-53.

3. Forcada-Guex M, Pierrehumbert B, Borghini A, Moessinger A. MullerNix C. Early dyadic patterns of mother-infant interactions and outcomes of prematurity at 18 months. Pediatrics. 2006;118(1):107-14.

4. Hollywood M, Hollywood E. The lived experiences of fathers of a premature baby on a neonatal intensive care unit. J Neonatal Nurs. 2011;17(1):32-40.

5. Benzies KM, Magill-Evans J, Hayden KA, Ballantyne M. Key components of early intervention programs for preterm infants and their parents: a systematic review and meta-analysis. BMC Pregnancy Childbirth. 2013;13(Suppl 1):1-15.

6. Cox SM, Hopkins J, Sydney LH. Attachment in preterm infants and their mothers: Neonatal risk status and maternal representations. Infant Ment Health J. 2000;21(6):464-80. 
7. Tiedje LB, Darling-Fisher C. Promoting father-friendly healthcare. MCN: The American Journal of Maternal Child Nursing. 2003;28(6):350-7.

8. Solís-Cámara RP, Bavolek SJ. Manual del Inventario de Paternidad para Adultos y Adolescentes (IPAA). Park City (Utah): Family Development Resources; 1995.

9. Resolución 008430/1993 del 4 de octubre, por la cual se establecen las normas científicas, técnicas y administrativas para la investigación en salud [internet]. Disponible en: http://www.minproteccionsocial.gov.co/VBeContent/library/documents/DocNewsNo267711.pdf

10. Consejo de Organizaciones Internacionales de las Ciencias Médicas. Pautas éticas internacionales para la investigación biomédica en seres humanos preparadas por el Consejo de Organizaciones Internacionales de las Ciencias Médicas (CIOMS) en colaboración con la Organización Mundial de la Salud [internet]. Chile; 2003. Disponible en: http://www. cioms.ch/publications/guidelines/pautas_eticas_internacionales.htm

11. Theunissen HC, Vogels AG, Reijneveld SA. Punishment and Reward in Parental Discipline for Children Aged 5 to 6 Years: Prevalence and Groups at Risk. Acad Pediatr. 2015;15(1):96-102.

12. Cho J, Holditch-Davis D, Belyea M. Gender, ethnicity, and the interactions of prematurely born children and their mothers. J Pediatr Nurs. 2004;19(3):163-75.

13. Khoury-Kassabri M. Attitudes of Arab and Jewish mothers towards punitive and non $\square$ punitive discipline methods. Child Fam Soc Work. 2010;15(2):135-44.

14. Taylor CA, Guterman NB, Lee SJ, Rathouz PJ. Intimate partner violence, maternal stress, nativity, and risk for maternal maltreatment of young children. Am J Public Health. 2009;99(1):175-83.

15. Holden GW, Brown AS, Baldwin AS, CroftCaderao K. Research findings can change attitudes about corporal punishment. Child Abuse Negl. 2014;38(5):902-8.

16. Casanueva C, Martin S, Runyan DK, Barth RP, Bradley RH. Parenting services for mothers involved with child protective services: Do they change maternal parenting and spanking behaviors with young children? Child Youth Serv Rev. 2008;30(8):861-78.

17. Evans T, Whittingham K, Sanders M, Colditz P, Boyd RN. Are parenting interventions effective in improving the relationship between mothers and their preterm infants? Infant Behav Dev. 2014;37(2):131-54.

18. Spielman V, Taubman-Ben-Ari O. Parental self-efficacy and stress-related growth in the transition to parenthood: a comparison between parents of pre- and full-term babies. Health Soc Work. 2009;34(3):201-12.

19. Carbonell OA, Plata SJ, Peña PA, Cristo M, Posada G. Calidad de cuidado materno: una comparación entre bebés prematuros en cuidado madre canguro y bebés a término en cuidado regular. Univ Psychol. 2010;9(3):773-85.

20. Nordhov SM, Kaaresen PI, Rønning JA, Ulvund SE, Dahl LB. A randomized study of the impact of a sensitizing intervention on the 
child-rearing attitudes of parents of low birth weight preterm infants. Scand J Psychol. 2010;51(5):385-91.

21. Rossman B, Greene MM, Meier PP. The role of peer support in the development of maternal identity for "NICU Moms". J Obstet Gynecol Neonatal Nurs. 2015;44(1):3-16.

22. Bialoskurski M, Cox CL, Hayes JA. The nature of attachment in a neonatal intensive care unit. J Perinat Neonatal Nurs. 1999;13(1):66-77.

23. Dhayanandhan B, Bohr Y, Connolly J. Developmental task attainment and child abuse potential in at-risk adolescent mothers. J Child Fam Stud. 2015;24(7):1987-98.

24. Hidalgo J, Hidalgo M. Madres adultas y madres adolescentes: un análisis comparativo de las interacciones que mantienen con sus bebés. Apunt Psicol. 2002;20(2):243-56.

25. Garrido MC, Marchán M. Adopción del rol materno en madres adolescentes primerizas según grupo de convivencia. Rev Psicol. 2011;13(1):11-28.

26. Araque S, Ariza NE, Valderrama ML. Estrategia educativa para el cuidado domiciliario de los bebés prematuros: madres usuarias del programa madre canguro de Tunja, Colombia. Rev Cuid. 2013;4(1):467-74.

27. López ME. Sentimientos de las madres con hijos prematuros hospitalizados. Revista In Crescendo-Ciencias de la Salud. 2014;1(2):263-70.

28. Villamizar B, Vargas C, Rueda E. Madres descubriendo el amor incondicional en el proceso adaptativo de hospitalización de su bebé prematuro. Rev Cuid. 2014;5(2):782-91. 